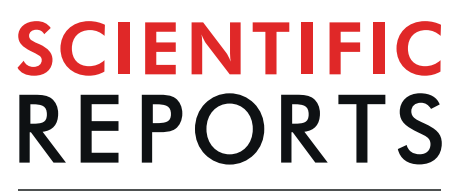

natureresearch

\title{
Elemental $\mathrm{Zn}$ and its Binding Protein Zinc- $\alpha 2$-Glycoprotein are Elevated in HPV-Positive Oropharyngeal Squamous Cell Carcinoma
}

\author{
Kate Poropatich ${ }^{1,2,3^{*}}$, Tatjana Paunesku ${ }^{3}{ }^{3}$, Alia Zander ${ }^{3}$, Brian Wray ${ }^{4}$, Matthew Schipma ${ }^{4}$, \\ Prarthana Dalal ${ }^{1}$, Mark Agulnik ${ }^{2,5}$, Si Chen ${ }^{6}$, Barry Lai ${ }^{6}$, Olga Antipova ${ }^{6}$, Evan Maxey ${ }^{6}$, \\ Koshonna Brown ${ }^{3}$, Michael Beau Wanzer ${ }^{3}$, Demirkan Gursel ${ }^{7}$, Hanli Fan ${ }^{7}$, Alfred Rademaker ${ }^{8}$, \\ Gayle E. Woloschak ${ }^{2,3}$ \& Bharat B. Mittal ${ }^{2,3}$
}

Human papillomavirus (HPV)-positive head and neck squamous cell carcinoma (HNSCC) is biologically distinct from HPV-negative HNSCC. Outside of HPV-status, few tumor-intrinsic variables have been identified that correlate to improved survival. As part of exploratory analysis into the trace elemental composition of oropharyngeal squamous cell carcinoma (OPSCC), we performed elemental quanitification by X-ray fluorescence microscopy (XFM) on a small cohort $(n=32)$ of patients with HPVpositive and -negative OPSCC and identified in HPV-positive cases increased zinc $(\mathrm{Zn})$ concentrations in tumor tissue relative to normal tissue. Subsequent immunohistochemistry of six Zn-binding proteinszinc- $\alpha 2$-glycoprotein (AZGP1), Lipocalin-1, Albumin, S100A7, S100A8 and S100A9-revealed that only AZGP1 expression significantly correlated to HPV-status $(p<0.001)$ and was also increased in tumor relative to normal tissue from HPV-positive OPSCC tumor samples. AZGP1 protein expression in our cohort significantly correlated to a prolonged recurrence-free survival $(p=0.029)$, similar to HNSCC cases from the TCGA $(n=499)$, where highest AZGP1 mRNA levels correlated to improved overall survival $(p=0.023)$. By showing for the first time that HPV-positive OPSCC patients have increased intratumoral Zn levels and AZGP1 expression, we identify possible positive prognostic biomarkers in HNSCC as well as possible mechanisms of increased sensitivity to chemoradiation in HPV-positive OPSCC.

Human papillomavirus (HPV)-positive oropharyngeal squamous cell carcinoma (OPSCC) is biologically and clinically distinct from HPV-negative OPSCC and HPV status is a well-established favorable prognostic variable $^{1}$. The continuously rising number of patients presenting with HPV-positive OPSCC in the United States has eclipsed the number of HPV-negative OPSCC ${ }^{1-3}$. Increased evidence demonstrates that even within HPV-positive head and neck squamous cell carcinoma (HNSCC), there are divergent clinical outcomes based on factors such as patient smoking history ${ }^{4}$. Immune-related tumor-extrinsic variables have been identified in HPV-positive OPSCC, including some by our group ${ }^{5,6}$, such as the phenotypic and morphologic features of tumor infiltrating lymphocytes. With regards to tumor-intrinsic variables, sex hormone overexpression and gene silencing through promoter methylation ${ }^{7,8}$ have recently been correlated to improved survival in HPV-positive OPSCC.

${ }^{1}$ Department of Pathology, Northwestern University Feinberg School of Medicine, Chicago, IL, 60611, USA. ${ }^{2}$ Robert H Lurie Comprehensive Cancer Center, Northwestern University Feinberg School of Medicine, Chicago, IL, USA. ${ }^{3}$ Department of Radiation Oncology, Northwestern University Feinberg School of Medicine, Chicago, IL, USA. ${ }^{4}$ Center for Genetic Medicine, Northwestern University Feinberg School of Medicine, Chicago, IL, USA. ${ }^{5}$ Division of Hematology and Oncology, Northwestern University Feinberg School of Medicine, Chicago, IL, USA. ${ }^{2}$ X-ray Science Division, Advanced Photon Source, Argonne National Laboratory, 9700 South Cass Avenue, Argonne, IL, 60439, USA. ${ }^{7}$ Northwestern University Pathology Core Facility, Robert H Lurie Comprehensive Cancer Center, Northwestern University Feinberg School of Medicine, Chicago, IL, USA. ${ }^{8}$ Department of Preventive Medicine, Northwestern University Feinberg School of Medicine, Chicago, IL, USA. *email: kate.poropatich@northwestern.edu 
As cofactors for multiple enzymes and proteins, essential trace elements-iron $(\mathrm{Fe})$, copper $(\mathrm{Cu})$, cobalt $(\mathrm{Co})$, manganese $(\mathrm{Mn})$ and zinc $(\mathrm{Zn})$-are required for normal biological functions ${ }^{9}$. Elevated levels of some elements such as $\mathrm{Fe}, \mathrm{Cu}$ and $\mathrm{Zn}$ have also been detected in the serum and tumor tissue from patients with different types of cancers ${ }^{10-14}$. At the same time, ample evidence indicates that different types of aggressive hormonally-driven human cancers (i.e. breast, prostate and ovarian) have low intratumoral $\mathrm{Zn}$ levels relative to normal tissue ${ }^{15-17}$. $\mathrm{Zn}$ exists largely bound to proteins and one type of $\mathrm{Zn}$-binding proteins are metallothioneins, which are involved in $\mathrm{Zn}$ metabolism and have been implicated in chemoradiosensitivity and restoration of mutant p53 in tumor cells ${ }^{18-23}$. The analysis of trace elements in HPV-positive OPSCC may assist in better understanding their intrinsic enhanced sensitivity to chemoradiation.

In this study, we utilize elemental mapping of a small cohort of clinically-matched HPV-positive and negative OPSCC cases $(n=32)$ by X-ray fluorescence microscopy $(X F M)$ and demonstrate $\mathrm{Zn}$ is increased in HPV-positive OPSCC tumor tissue relative to matched normal squamous mucosa. By performing a targeted analysis of the expression of six Zn-binding proteins-Lipocalin-1, zinc- $\alpha 2$-glycoprotein (AZGP1), albumin, S100A7, S100A8 and S100A9-in tumors from OPSCC patients $(n=68)$, we reveal AZGP1 is selectively overexpressed in a subpopulation of HPV-positive OPSCC patients and that its expression is an independent predictor of patient survival in this patient population.

\section{Results}

Patient population. A total of 75 cases of OPSCC with sufficient tissue for further testing were identified (Supplemental Fig. 1), of which 48 were HPV-positive. The majority of patients were male and the median age at the time of diagnosis was 58 years (Supplemental Table 1). While HPV-positive patients were more likely to be never-smokers compared to HPV-negative patients, this was not significant. Among different treatment modalities, combination surgery and chemoradiation was the most common, accounting for approximately half of all the patients in the cohort (Supplemental Table 1). As expected, HPV-negative OPSCC patients were more likely to have a recurrence, accounting for $59 \%$ of all recurrences (10/17), and the recurrence free survival (RFS) was significantly longer in HPV-positive patients compared to HPV-negative patients (3.71 years vs. 2.51 years, $\mathrm{p}=0.001)$.

Elemental measurement and mapping by XFM. Among the 16 elements mapped by low-resolution XFM scanning of tumor and histologically normal adjacent mucosa in OPSCC cases $(n=32)$, the quality of data was high and suitable for analysis for the following eight elements: $\mathrm{P}, \mathrm{S}, \mathrm{K}, \mathrm{Cl}, \mathrm{Ca}, \mathrm{Fe}, \mathrm{Cu}$ and $\mathrm{Zn}$. Given that $\mathrm{K}$ and $\mathrm{Cl}$ measurements are often inaccurate in formalin-fixed paraffin-embedded (FFPE) samples ${ }^{24}$, we focused on Ca, $\mathrm{Cu}, \mathrm{Fe}, \mathrm{P}$ and $\mathrm{Zn}$ in HPV-positive and -negative patient pairs matched for different clinical features (Methods), using $\mathrm{S}$ to normalize the data (Methods, Fig. 1). Among these elements, normalized $\mathrm{P}$ and $\mathrm{Zn}$ concentrations were significantly elevated in tumor tissue relative to matched adjacent normal mucosa from HPV-positive but not HPV-negative OPSCC cases $(\mathrm{p}=0.0015$ and 0.0228 , respectively) (Fig. 2a). We then compared elemental tumor-to-normal ratios for $16 \mathrm{HPV}$-positive/HPV-negative patient pairs. Whereas Fe tumor:normal ratios were $>1$ for both HPV-positive and -negative OPSCC cases, only HPV-positive patients had ratios $>1$ for both $\mathrm{P}(\mathrm{p}=0.0263)$ and $\mathrm{Zn}(\mathrm{p}=0.0204)$ in the tumor tissue relative to normal mucosa (Fig. 2b). As differences in $\mathrm{P}$ concentrations may be in part ascribed to factors such as the increased nuclear content of HPV-positive tumors and aneuploidy nature of the tumors, which can increase DNA content ${ }^{25}$, we decided to focus our investigation on differences in $\mathrm{Zn}$ content between the two groups, especially in light of the fact that $\mathrm{Zn}$ has been shown to synergize with chemoradiation to induce sensitivity in different human tumor models ${ }^{19-21,23}$.

In order to perform a more in-depth analysis of elemental differences between HPV-positive and -negative samples, we scanned one of the matched HPV-positive and -negative patient pairs with medium-resolution XFM (Fig. 2c). Total quantities of elements (picograms) were determined from a region of interest (ROI) of 40 tumor cells per case (Supplemental Fig. 2, Supplemental Table 2). In this evaluation, the HPV-positive patient had approximately twice as high a quantity of $\mathrm{Zn}$ (1.67 picograms) compared to the HPV-negative patient $(0.902$ picogram).

Identification of Zn-binding proteins by mass spectrometry. FFPE tumor tissues from an $\mathrm{HPV}$-positive and -negative OPSCC patient pair were subjected to exploratory proteomic analysis. Among 276 proteins identified from laser-captured microdissection of tumor tissue, 47 unique proteins were present in the tumor from the HPV-positive patient, 36 were present in the tumor from the HPV-negative patient and the remaining 193 proteins were shared amongst both patient tumor samples (Supplemental Table 3). Among the most frequently detected proteins in both tumor samples were proteins indicative of epithelial tumor cell origin, including type I/type II keratins and actin.

As $\mathrm{Zn}$ had been identified from our XFM data as an element increased in the tumor from HPV-positive OPSCC patients, we further interrogated the 276 proteins for those that were $\mathrm{Zn}$-binding in the tumor samples from either patient (Supplemental Table 3). Of these nine Zn-binding proteins, six were found in both tumor samples (AZGP1, Serum albumin, S100A8/Calgranulin-A, S100A9/Calgranulin-B, S100A7/Psoriasin, and Ubiquitin-40S ribosomal protein S27a), one was found only in the HPV-positive tumor sample (Lipocalin-1) and two were found in the HPV-negative tumor sample (Collagen alpha-1(XVIII) chain and Carbonic anhydrase 1).

Zn-binding protein AZGP1 is elevated in subset of HPV-positive OPSCC cases. We measured the expression of six different $\mathrm{Zn}$-binding proteins in tissue microarrays (TMAs) from OPSCC patients by IHC in matched tumor and normal tissue, including: Lipocalin-1 $(n=63)$, AZGP1 $(n=68)$, albumin $(n=26)$, S100A7 $(n=53)$, S100A7 $(n=53)$, S100A8 $(n=51)$ and S100A9 $(n=50)$. We used a graded scoring system 

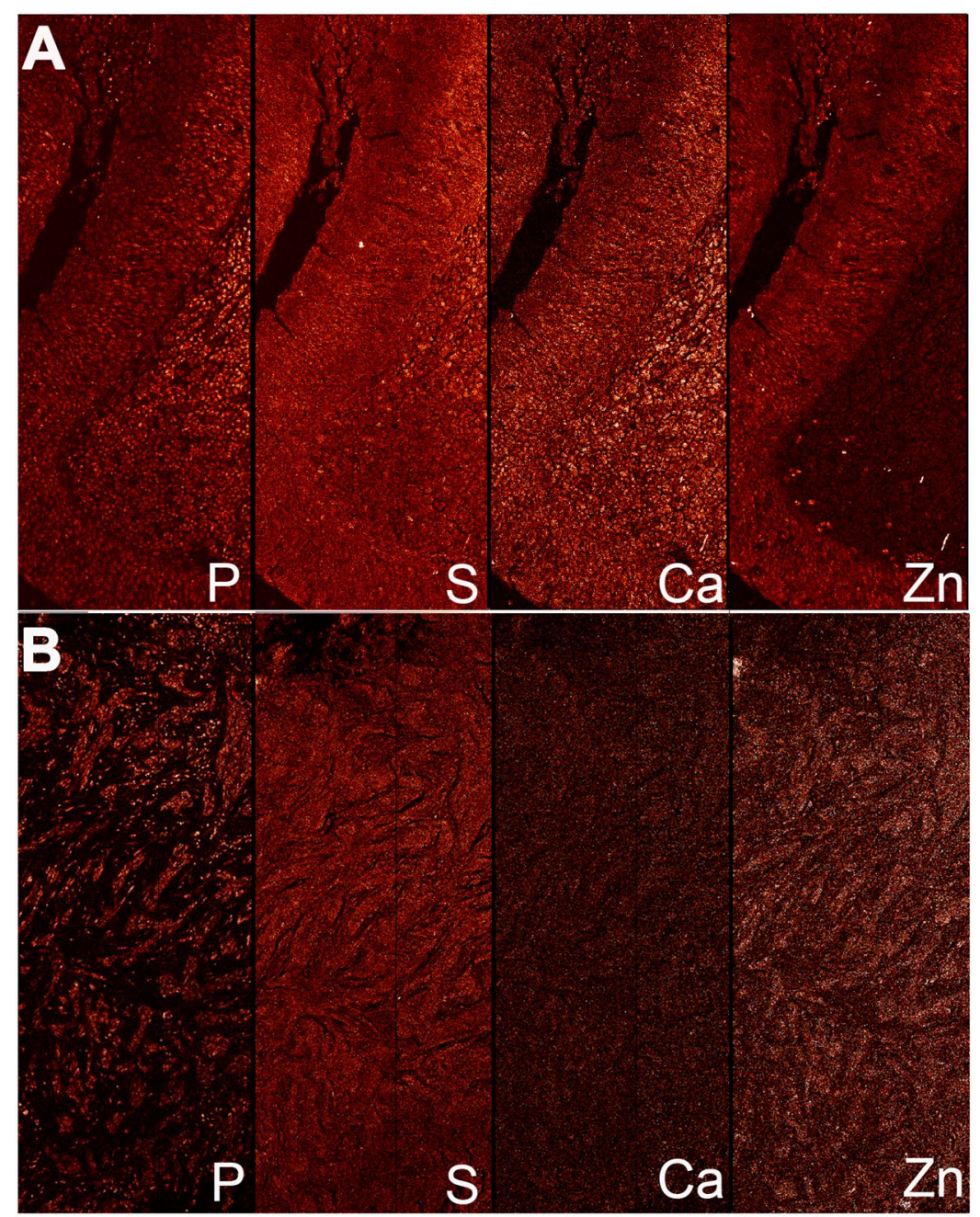

Figure 1. XFM elemental maps for a matched OPSCC patient pair. Medium resolution (beam focused to $0.5 \mu \mathrm{m}$ spot) images were collected from formalin-fixed whole-tissue sections of tumor from patients with OPSCC ( $\mathrm{n}=32,16 \mathrm{HPV}$-positive, $16 \mathrm{HPV}$-negative). Shown are the elemental maps for P, S (reference element for normalization), $\mathrm{Fe}, \mathrm{Ca}$ and $\mathrm{Zn}$ in the tumor from a matched HPV-positive OPSCC (A) and HPV-negative OPSCC (B) patient pair.

for staining intensity (low $=1$, moderate $=2$ and high $=3$ ) in tumor and matched normal squamous mucosa. Among the six Zn-binding proteins, AZGP1 was the only protein with significant differential expression levels amongst HPV-positive and HPV-negative samples, with higher median expression in tumor relative to normal in HPV-positive cases compared to HPV-negative cases (Fig. 3, Supplemental Table 4). An opposite pattern was evident for HPV-negative cases in this cohort-tumor AZGP1 staining was reduced relative to adjacent normal mucosa. In contrast, median Lipocalin-1 staining was actually higher in the tumor of HPV-negative cases versus HPV-positive cases, albeit not statistically significant.

Correlation analysis revealed that HPV status significantly positively correlated to AZGP1 $(\mathrm{p}=0.0001)$ but not Lipocalin-1 staining intensities; 13 of 14 (93\%) of AZGP1-high cases were HPV-positive and among AZGP1-low cases $(n=15)$, two-third $(n=10)$ were HPV-negative (Fig. $4 a-c)$. Additionally, Lipocalin-1 was significantly inversely correlated to AZGP1 expression $(\mathrm{p}=0.001)$. Collectively, our findings suggest that in HPV-positive OPSCC, AZGP1 is overexpressed in a subset-approximately one-in-three-and that its protein expression may be downregulated in HPV-negative OPSCC patients.

Patient survival is positively correlated to AZGP1 expression. We chose to further focus on Lipocalin-1 and AZGP1 protein expression for patient survival analysis based on our findings of inverse expression trends in our patient cohort. As AZGP1 has been demonstrated to have anti-tumor activity and improved clinical outcome in cancer patients, we hypothesized its overexpression may portend improved patient survival [26,27]. Among our cohort cases with available survival data (Supplemental Table 1), we found that overexpression of AZGP1 significantly correlated to a longer RFS and that reduced expression correlated to a shorter RFS ( $p=0.029$ ); there were no recurrences in the AZGP-1 high group compared to nearly one-in-two recurrences in the AZGP1-low group, respectively (Table 1, Fig. 4d). In contrast, no survival benefit was present in our cohort of OPSCC patients based on Lipocalin-1 expression patterns. 
A.
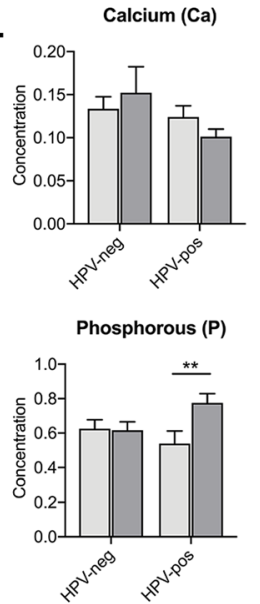

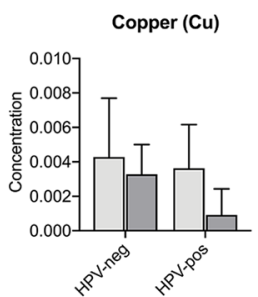

Zinc (Zn)

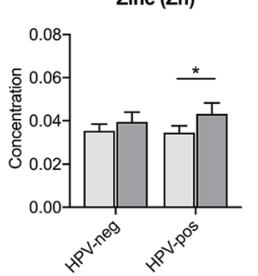

B. Elemental Tumor:Normal Ratios

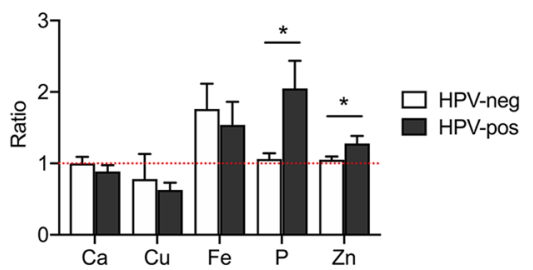

C.
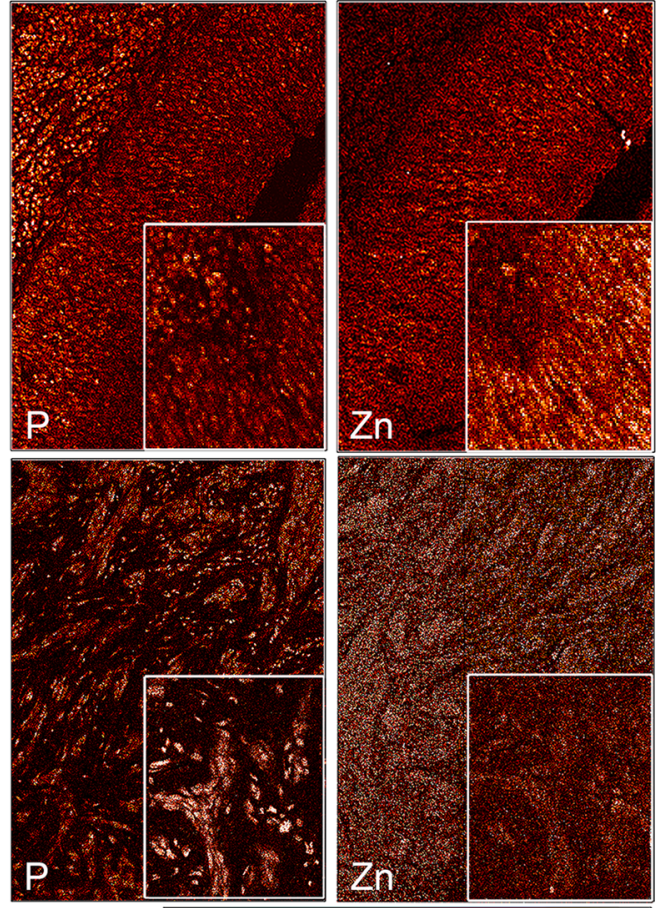

Figure 2. Quantified elemental concentrations in HPV-positive and -negative OPSCC cases. (A) Elemental concentrations for $\mathrm{Ca}, \mathrm{Cu}, \mathrm{Fe}, \mathrm{P}$ and $\mathrm{Zn}$ in tumor and adjacent normal mucosa from OPSCC patients $(\mathrm{n}=32)$. Data from scans were calibrated and per pixel counts were converted to elemental concentrations $\left(\mu \mathrm{g} / \mathrm{cm}^{2}\right)$ (Methods). Final measurement of elemental concentrations were normalized relative to sulfur. (B) Ratios of tumor-to-normal for individual samples were calculated for $\mathrm{Ca}, \mathrm{Cu}, \mathrm{Fe}, \mathrm{P}$ and $\mathrm{Zn}$ for $16 \mathrm{HPV}$-positive and HPV-negative patient pairs (Methods). (C) Medium resolution XFM tumor elemental maps for P and $\mathrm{Zn}$ in a clinically-matched HPV-positive (top) and HPV-negative (bottom) OPSCC patient pair. These detailed maps are representative of larger tumor region scans shown in Fig. 1. For elemental quantification from this patient pair, see Supplemental Table 2. Bar graph data are mean \pm s.e.m; statistical significance evaluated by a paired Student's T test $(\mathrm{a}, \mathrm{b}) .{ }^{*} P<0.05 ; * * P<0.01$.

In order to determine whether the improved outcome of AZGP1 expression was dependent on other variables, covariates were addressed using the accelerated failure time (AFT) model (Table 1). Due to the fact that AZGP1 expression was almost exclusively in HPV-positive OPSCC, HPV status could not be used as a covariate in multivariate analysis. When adjusting for covariates, however, such as smoking status or treatment plan, AZGP1 protein expression positively correlated to improved patient survival.

When survival analysis was extended to The Cancer Genome Atlas (TCGA) to interrogate the relationship of AZGP1 and LCN1 mRNA transcript levels with HNSCC patient survival $(\mathrm{n}=499)$, similar patterns were evident for both genes with their protein counterparts (Fig. 4e). Overexpression of $A Z G P 1 \mathrm{mRNA}(\mathrm{n}=125$, based on top quartile expression) correlated to longer overall survival in HNSCC patients, although low AZGP1 mRNA levels $(\mathrm{n}=124$, based on bottom quartile expression) did not portend a worse overall outcome. No significant difference in overall survival was present based on $L C N 1$ expression levels.

AZGP1 mRNA levels correlate to HPV-integration status. Recent work has characterized the HPV-host status (integrated vs. episomal) in HNSCC, including OPSCC, from RNA-sequencing (RNA-seq) data in TCGA and Gene Expression Omnibus (GEO) ${ }^{28}$. We performed an analysis of AZGP1 mRNA transcript levels from RNA-seq data available in the databases for HPV-positive and HPV-negative OPSCC cases, including a total of 97 patients with OPSCC from TCGA $(n=80)$ and GEO portals $(n=17)^{28}$. The majority of cases were HPV-positive $(n=83)$ compared to HPV-negative. While total mean levels were higher in the HPV-negative OPSCC cases compared to HPV-positive OPSCC cases, this was not statistically significant by ANOVA analysis (F value: $2.643, \mathrm{p}=0.0764$ ). Among the HPV-positive OPSCC cases, approximately two-thirds had integrated HPV $(\mathrm{n}=50)$ and one-third had episomal HPV $(\mathrm{n}=33)$. HPV-integrated OPSCC cases had significantly higher levels of AZGP1 mRNA transcripts compared to OPSCC cases with episomal HPV (Fig. 4f, p=0.046, Wilcoxon rank test) (Fig. 4f).

\section{Discussion}

The elemental and protein-based analysis of OPSCC patients presented here is a coordinated strategy to develop new understandings of the pathogenesis of OPSCC. This is the first attempt to characterize the composition of OPSCC tumor tissue by trace elements using XFM and to demonstrate that Zn-binding protein AZGP1 is a potential biomarker for positive prognosis in HNSCC. Our findings add to other work that also demonstrates that 


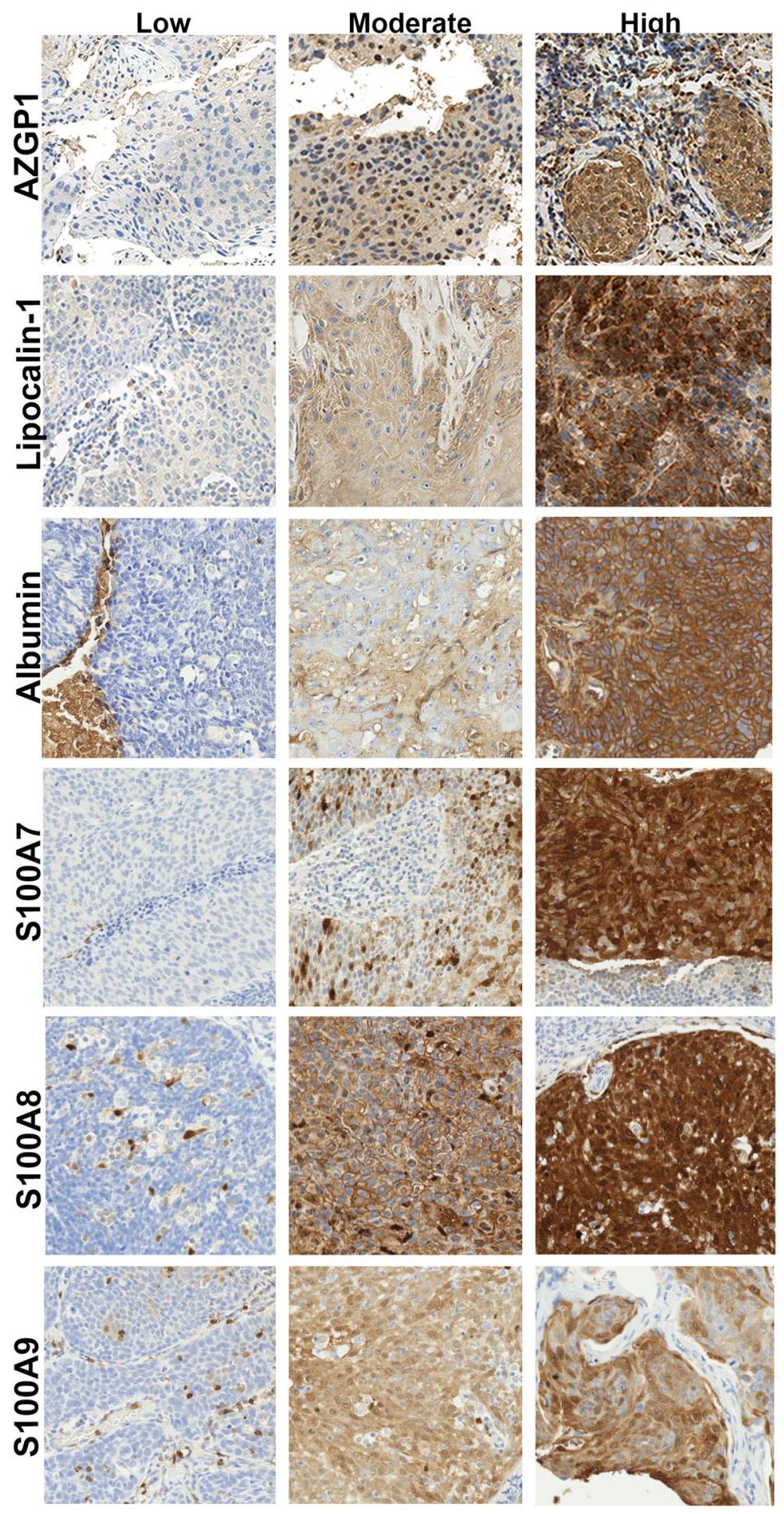

Figure 3. Zn-binding protein immunohistochemical staining in OPSCC. Examples from nanozoomer digitized scans (20X magnification) of low, moderate and high cytoplasmic staining of OPSCC tumor tissue for the $\mathrm{Zn}$ binding proteins AZGP1, Lipocalin-1, serum Albumin, S100A7, S100A8 and S100A9.

patient outcome in HPV-positive OPSCC is affected by variables such as smoking status, hormonal status, gene expression and methylation patterns and HPV integration status ${ }^{4,7,8,28}$.

Studies utilizing XFM have shown distinct patterns in the tumor microenvironment (TME) of different human cancers ${ }^{29,30}$. In this study we found that the normalized tumor-to-normal $\mathrm{P}$ and $\mathrm{Zn}$ ratios were elevated in HPV-positive OPSCC patients compared to the ratios for HPV-negative patients. In different aggressive human solid tumor models (i.e. breast, prostate and ovarian), intratumoral Zn levels are low relative to normal tissue ${ }^{15-17}$. As an antioxidant, $\mathrm{Zn}$ has been studied as a tool to interrupt multiple carcinogenesis-related pathways ${ }^{31}$ and 
A.
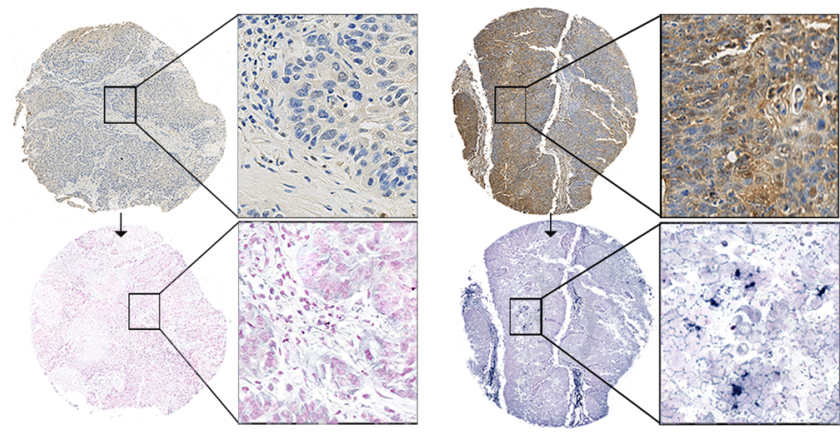

D. Lipocalin-1 (IHC, NMH)

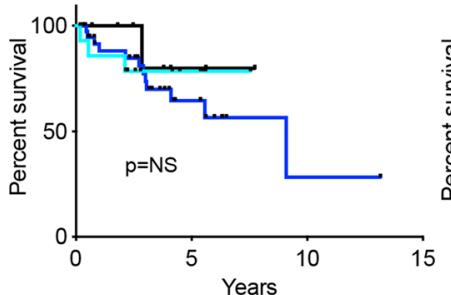

E.

LCN1 (mRNA, TCGA)

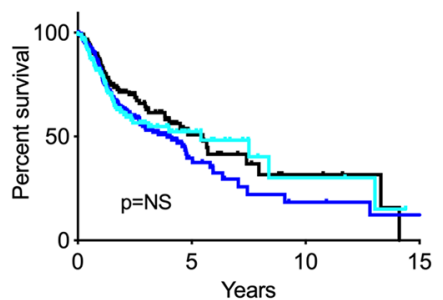

AZGP1 (IHC, NMH)

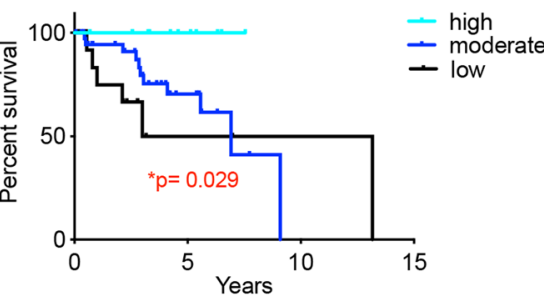

AZGP1 (mRNA, TCGA)

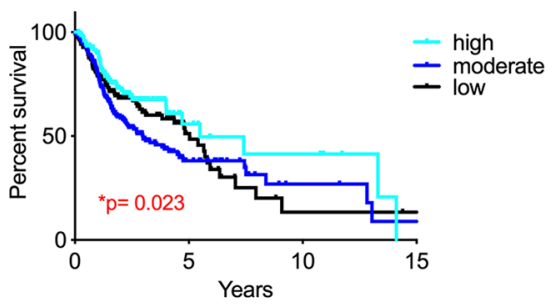

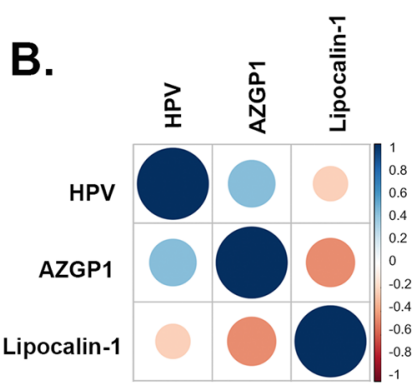

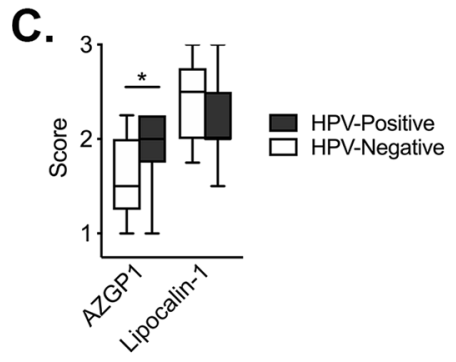

F.

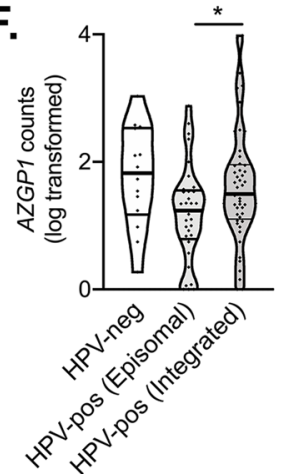

Figure 4. AZGP1 correlates to HPV-status and improved survival in OPSCC and HNSCC. (A) Comparison of AZGP1 immunohistochemical staining with HPV-status confirmed by in situ hybridization staining in HNSCC; Strong AZGP1 expression is detected in HPV-positive tumor tissue (right) and absent in HPVnegative tumor tissue (left) (B,C) A correlation matrix for HPV, AZGP1 and Lipocalin-1 in which HPV status significantly positively correlated to AZGP1 protein expression. Pearson correlation analysis. Box-and-whiskers plot shows comparison of HPV-positive and -negative OPSCC staining levels $($ low $=1$, moderate $=2$, high $=3$ ) for AZGP1 and Lipocalin-1. Student's T test. (D) Kaplan-Meier analysis of recurrence-free survival (RFS) in patients with low, moderate and high IHC levels for AZGP1 $(n=66)$ and Lipocalin-1 $(n=63)$. Overexpression of AZGP1 protein in OPSCC corresponded to significantly prolonged RFS (log-rank, $p=0.029)$. (E) Survival analysis in HNSCC patients $(\mathrm{n}=499)$ from the TCGA with $L C N 1$ and AZGP1 mRNA levels stratified as high, moderate and low. AZGP1-high cases have a significantly longer overall survival (log-rank, $\mathrm{p}=0.023)$. (F) RNA-seq analysis of OPSCC cases from TCGA and GEO comparing AZGP1 mRNA levels in HPV-negative, HPV-positive (integrated) and HPV-positive (episomal) OPSCC patients. Mean AZGP1 mRNA counts for the HPV-integrated OPSCC cases were higher than in HPV-positive OPSCC with episomal HPV $(\mathrm{p}=0.04637)$; Wilcoxon rank test. Data is shown as a violin plot (bold line at median and thin lines at upper and lower quartiles). $* P<0.05$.

administration of exogenous $\mathrm{Zn}$ has been implicated as a strategy to increase chemoradiosensitivity ${ }^{18-23}$. In HNSCC, the reduction of systemic Zn levels is correlated to aggressive tumor development-a pilot human longitudinal study of patients with advanced HNSCC revealed that in the immediate timeframe before cancer-related death, $\mathrm{Zn}$ levels drop from baseline in the majority of patients ${ }^{32}$. Subsequently, clinical trials of oral HNSCC have incorporated oral $\mathrm{Zn}$ therapy into their protocols as adjuvant therapy ${ }^{33}$.

Zn stabilizes the structurally complex DNA-binding domain of the tumor suppressor gene p53 and has also been shown to rescue wild-type $\mathrm{p} 53$ activity in mutant $\mathrm{p} 53$ by re-establishing chemosensitivity in p53-mutated cell lines ${ }^{21}$. While entirely speculative, our finding of increased intratumoral Zn content in HPV-positive OPSCC cases could provide justification for the intrinsic chemoradiosensitivity of this patient population and offer insight into ways to improve the clinical outcome of patients with HPV-negative HNSCC, who frequently harbor mutant p53.

Unlike P, which is predominantly associated with nucleic acids, the vast majority of intra- and extracellular $\mathrm{Zn}$ is bound to proteins, serving either structural or catalytic roles, collectively referred to as the 'zinc proteome ${ }^{34}$. While we could not conduct a complete survey of all possible $\mathrm{Zn}$ binding proteins, we tested for the expression 


\begin{tabular}{|c|c|c|c|c|c|c|c|c|c|c|c|c|c|}
\hline \multirow{3}{*}{\multicolumn{4}{|c|}{ Kaplan-Meier Estimate }} & \multicolumn{10}{|l|}{ AFT Model } \\
\hline & & & & \multirow{2}{*}{\multicolumn{2}{|c|}{ Univariate }} & \multicolumn{8}{|c|}{ Multivariate } \\
\hline & & & & & & \multicolumn{2}{|l|}{ HPV-status } & \multicolumn{2}{|l|}{ T stage ${ }^{\mathrm{a}}$} & \multicolumn{2}{|c|}{ Smoking-status ${ }^{\mathrm{b}}$} & \multicolumn{2}{|l|}{ Treatment $t^{c}$} \\
\hline Protein & $\begin{array}{l}\text { No.Patients (No. } \\
\text { Events) }\end{array}$ & $\begin{array}{l}\text { Survival } \\
\text { Proportion }\end{array}$ & $\begin{array}{l}\text { Log-rank } P \\
\text { value }\end{array}$ & \begin{tabular}{|l|} 
TR \\
$(95 \%$ CI $)$
\end{tabular} & $P$ & \begin{tabular}{|l} 
TR \\
$(95 \%$ CI $)$
\end{tabular} & $P$ & \begin{tabular}{|l|} 
TR \\
$(95 \%$ CI $)$
\end{tabular} & $P$ & \begin{tabular}{|l|} 
TR \\
$(95 \% \mathrm{CI})$
\end{tabular} & $P$ & \begin{tabular}{|l|} 
TR \\
$(95 \% \mathrm{CI})$
\end{tabular} & $P$ \\
\hline AZGP1 & \begin{tabular}{|l|}
$66(16)$ \\
High =12 (0) \\
Medium = 39 (10) \\
Low =15 (6) \\
\end{tabular} & \begin{tabular}{|l}
100 \\
61.72 \\
50.0
\end{tabular} & 0.029 & $\begin{array}{l}2.24 \\
(2.20,2.28)\end{array}$ & $<0.001$ & $\begin{array}{l}1.22 \\
(0.29,5.05)\end{array}$ & NS & $\begin{array}{l}2.50 \\
(0.90,6.93)\end{array}$ & 0.078 & $\begin{array}{l}2.32 \\
(2.26,2.38)\end{array}$ & $<0.001$ & $\begin{array}{l}2.879 \\
(2.85,2.91)\end{array}$ & $<0.001$ \\
\hline \multirow{4}{*}{ Lipocalin-1 } & $\begin{array}{l}63(16) \\
\text { High }=15(3)\end{array}$ & 78.57 & \multirow{4}{*}{ NS } & \multirow{4}{*}{$\begin{array}{l}0.78 \\
(0.15,4.02)\end{array}$} & \multirow{4}{*}{ NS } & \multirow{4}{*}{$\begin{array}{l}1.38 \\
(0.33,5.85)\end{array}$} & \multirow{4}{*}{ NS } & \multirow{4}{*}{\begin{tabular}{|l}
0.740 \\
$(0.099,5.54)$
\end{tabular}} & \multirow{4}{*}{ NS } & \multirow{4}{*}{$\begin{array}{l}0.77(0.13, \\
4.52)\end{array}$} & \multirow{4}{*}{ NS } & \multirow{4}{*}{$\begin{array}{l}0.897 \\
(0.07,10.8)\end{array}$} & \multirow{4}{*}{ NS } \\
\hline & Medium $=40(12)$ & 27.13 & & & & & & & & & & & \\
\hline & \multirow{2}{*}{ Low $=8(1)$} & 9 & & & & & & & & & & & \\
\hline & & 80.0 & & & & & & & & & & & \\
\hline
\end{tabular}

Table 1. Uni- and Multivariate Survival Analysis of AZGP1 and Lipocalin-1 in OPSCC cohort. Abbreviations: $\mathrm{AFT}=$ accelerated failure time, $\mathrm{TR}=$ time ratio, $\mathrm{CI}=$ confidence interval. ${ }^{\mathrm{a}}$ Defined as $\mathrm{T} 1$ (reference) versus $\mathrm{T} 2$ or T3/T4. Method $=$ Nelder-Mead and control $=$ list $($ fnscale $=2500) .{ }^{b}$ Defined as never-smoker versus any smoking history. ${ }^{\mathrm{c}}$ Defined as surgery with chemoradiation versus chemoradiation-only or surgery-only.

of six Zn-binding proteins in our OPSCC cohort that we initially detected in OPSCC by mass spectroscopyLipocalin-1, AZGP1, albumin, S100A7, S100A8 and S100A9-and we found that only AZGP1 expression was significantly higher in HPV-positive OPSCC tumor cells.

AZGP1 is a soluble $40-\mathrm{kDa}$ protein and major histocompatibility complex homolog and adipokine located on human chromosome 7q22.1. It is secreted by epithelial ductal cells of the salivary glands, breast, prostate and gastrointestinal tract ${ }^{35,36}$. In oral and buccal squamous epithelium, AZGP1 is intracellularly expressed in the mature layers of the stratified squamous epithelium ${ }^{37}$. AZGP1 expression may be related to HPV infection of human squamous epithelium - transcriptomic analysis of mature keratonicytes infected with HPV-16 demonstrated that AZGP1 mRNA is one of the six most-upregulated genes ${ }^{38}$. AZGP1 has one strong Zn-binding site and up to 15 weak $\mathrm{Zn}$-binding sites. The strong $\mathrm{Zn}$-binding site is located close to the lipid-binding helical groove that AZGP1 uses as Zn-binding ligands ${ }^{39}$. Zn, but no other divalent metals, induces the oligomerization of AZGP1 and is required for its functional activity ${ }^{40}$. While increased $\mathrm{Zn}$ and AZGP1 secretion into the serum have been found in different human epithelial malignancies, we found for the first time that both Zn and AZGP1 levels were increased in the cytoplasmic compartment of HPV-positive OPSCC tumor cells. Further work is needed to elucidate the co-localization of $\mathrm{Zn}$ with AZGP1 in the cytoplasm of tumor cells.

Abundant evidence demonstrates that the loss of expression of AZGP1-just like Zn-in tumor cells is a negative prognostic biomarker for different solid tumors, including breast, gastric, esophageal, soft tissue and prostate cancers. In these cancers, loss of AZGP1 protein was also associated with an increased likelihood of lymph node metastasis and advanced clinical stage ${ }^{26,41-45}$. In the TME, data supports the conclusion that AZGP1 could function as a tumor suppressor gene through inhibition of ERK signaling ${ }^{27}$.

From our exploratory protein expression analysis by IHC, we found that a subset of all OPSCC patients overexpressed AZGP1 protein and had a significantly longer RFS compared to patients with absent or low AZGP1 expression, which we corroborated with survival analysis of HNSCC patients in the TCGA. In comparison to previous literature that reports increased AZGP1 staining in oral cavity squamous cell carcinoma with histologic evidence of maturation (keratinization), the vast majority $(n=12 / 14,86 \%)$ of AZGP1-high OPSCC tumors in our cohort showed undifferentiated morphology (data not shown ${ }^{36}$. This is in-keeping with the typical morphologic features of HPV-associated HNSCC and reflects an important distinction in AZGP1 staining patterns in distinct anatomic locations of the head and neck.

In light of these protein expression results, we wanted to explore corresponding AZGP1 mRNA levels in HPV-positive and HPV-negative OPSCC patients. Our analysis of TCGA and GEO RNA-seq data for AZGP1 mRNA levels revealed that total mean AZGP1 mRNA transcrips were higher in HPV-negative OPSCC, though it is impossible to make conclusions from this based on the small patient size of TCGA HPV-negative OPSCC cases. If AZGP1 mRNA levels are indeed higher in HPV-negative OPSCC, our findings of its reduced expression at the protein level in our HPV-negative OPSCC, albeit also small in size and difficult to permit general conclusions, could be due to AZGP1 post-translational and/or epigenetic silencing through histone deacetylase (HDAC) activity, which is an established epigenetic silencing mechanism of AZGP1in different human cancers ${ }^{27,46}$.

While our methods did not allow us to segregate between episomal and integrated HPV-status in our OPSS cohort, we show that TCGA/GEEO cases with integrated HPV had significantly higher AZGP1 mRNA levels compared to those cases with episomal HPV. What these findings from RNA-seq mean for the protein expression, however, remains to be established. The HPV oncoproteins E6 and E7 encode Zn finger transcription activation domains comprised of cysteine residues that are required for their function ${ }^{47-49}$. Additionally, the HPV16 E5 protein blocks the negative regulation of $\mathrm{Zn}$ redistribution into host cell nucleoli through interactions with the $\mathrm{Zn}$ transporter complex made of proteins EVER1 and 2 and ZnT- $1^{50}$, resulting in increased cell proliferation. Further investigation is warranted to determine if HPV-related proteins such as EVER2 contribute to our finding that $\mathrm{Zn}$ is elevated in HPV-positive tumor cells. We speculate other Zn-binding proteins that are not encoded by the HPV genome, such as AZGP1, also contribute to increased detectable Zn levels in OPSCC cases.

This study is intended to draw previously unrecognized correlations between HPV-status in OPSCC with intratumoral trace element content and the expression of metal-binding proteins and not to establish mechanisms 
for which one finding may be related to another. We believe that both Zn and AZGP1 are promising therapeutic targets in HNSCC, though their role in patient sensitivity to chemoradiation needs to be further studied in a larger population. Major limitations of this study include the relatively small cohort size and the robustness of the correlation of overall tumor $\mathrm{Zn}$ levels with specific $\mathrm{Zn}$ binding proteins. With respect to the former, the number of patients in this cohort is not of adequate size to correlate to the general population. We attempted to remedy this by extending our survival analysis of AZGP1 to the larger HNSCC population database available in TCGA. Also, due to lack of tissue in some cases, we were not able to achieve statistical significance for a correlation between AZGP1 expression and $\mathrm{Zn}$ levels on a per-case basis.

\section{Methods}

Specimens. All archived patient specimens for retrospective analysis were collected from patients diagnosed with OPSCC at Northwestern Memorial Hospital (NMH), Department of Pathology under a Northwestern University (NU) IRB-approved protocol. All experiments were performed in accordance with relevant guidelines and regulations. The need for informed consent was waived by the NU IRB. Cases were selected over a six-year period (2010-2016) based on the following criteria: a primary diagnosis of squamous cell carcinoma of the oropharynx with available p16 immunohistochemistry (IHC) results and adequate tissue for testing. Among all patients, $16 \mathrm{HPV}$-positive and HPV-negative patient pairs were created (A.R.) for XFM imaging using the following matching variables: age, smoking, alcohol use, tumor differentiation, tumor location, tumor stage and gender.

FFPE tissues selected for this study were used for construction of TMAs as three $1.5 \mathrm{~mm}$ cores per case, comprised of two tumor cores and one core of adjacent histologically normal squamous mucosa, verified by a pathologist (K.P.). Tonsil cores from healthy donors were included in selection for the TMA as staining controls. Five $\mu \mathrm{m}$ sections were cut from tissue and TMA blocks for staining with hematoxylin and eosin (H\&E) and for IHC. Tissue sections for XFM were placed on Ultralene membranes (SPEX Sample Prep, LLC, 15 Liberty St., Metuchen, NJ 08840, USA).

HPV detection. At the time of diagnostic work-up, all cases were stained for p16-the HPV surrogate marker ${ }^{51}$-and interpreted as positive when $>70 \%$ strong tumor nuclear and cytoplasmic staining was present. We also performed high-risk (HR) HPV testing on all cases using DNA and mRNA in situ hybridization (ISH). DNA ISH: ZytoFast ${ }^{\circledR}$ HPV High-Risk (HR) Types Probe specific for oncogenic E6/E7 from 15 HPV types, Cat. T-1140-400 combined with the Zytofast PLUS Implementation Kit, Cat. T-1061-40). mRNA ISH: RNAscope 2.0 HD Detection Kit (Brown, ACD, Cat. 322300). RNAscope testing used a probe against 18 high-risk (HR) HPV genotypes E6/E7 mRNA (RNAscope Probe HPV-HR18, ACD, Cat. 312591). Slides were processed according to the manufacturer's instructions; the bacterial gene $\mathrm{DapB}$ was used as a negative control and housekeeping gene POLR2A served as a positive control.

XFM imaging. XFM was done (T.P.) on FFPE whole-sections of tumor from patients with OPSCC $(\mathrm{n}=32,16$ HPV-pos, 16 HPV-neg; Supplemental Fig. 1) at the 8BM-B and 2ID-E beamlines at the Advanced Photon Source at Argonne National Laboratory as previously described ${ }^{52,53}$. At the $8 \mathrm{BMB}$ beamline, the beam was focused to a $30 \mu \mathrm{m}$ spot (low resolution) and at the beamline 2-ID-E the beam was focused to a $0.5 \mu \mathrm{m}$ spot (medium resolution). Spectra were collected with a SII Vortex ME4 4-element silicon drift detector (SII NanoTechnology USA, Northridge, CA). Tissue sections were scanned with step sizes equal or larger than beam spot sizes and dwell times of 100-500 milliseconds; their elemental content was quantified and mapped. Elemental concentrations were calibrated using thin film AXO standards (Applied X-ray Optics, Dresden, Germany) and the data were calibrated using MAPS software ${ }^{54}$. Per pixel counts were converted to elemental concentrations $\left(\mu \mathrm{g} / \mathrm{cm}^{2}\right)$.

Data for 16 elements were collected: $\mathrm{Zn}, \mathrm{Mn}, \mathrm{Fe}, \mathrm{Cu}, \mathrm{Co}$, phosphorous $(\mathrm{P})$, sulfur $(\mathrm{S})$, chlorine $(\mathrm{Cl})$, potassium $(\mathrm{K})$, calcium $(\mathrm{Ca})$, chromium $(\mathrm{Cr})$, nickel $(\mathrm{Ni})$, arsenic $(\mathrm{As})$, selenium $(\mathrm{Se})$, bromine $(\mathrm{Br})$ and mercury $(\mathrm{Hg})$. Concentrations of some of the elements $(\mathrm{Cr}, \mathrm{Mn}, \mathrm{Co}, \mathrm{Ni}, \mathrm{As}, \mathrm{Se}, \mathrm{Br}, \mathrm{Hg}$ ) fell within the error range and those elements were not used for final analysis. Final measurement of elemental concentrations was done as follows: for each selected region of interest (ROI), mean per pixel value background area concentration for each element was subtracted from elemental concentrations for tumor and adjacent histologically benign squamous mucosa. Finally, these values were normalized relative to sulfur. Sulfur was chosen for this purpose due to the fact that it has a uniform signal in proteins from FFPE tissue, serving as a proxy for tissue density ${ }^{55}$. Normalizing to sulfur was also necessary because cell density and per cell sizes in ROI varied.

Laser capture microdissection and mass spectroscopy. For two patients, FFPE tumor sections eight $\mu \mathrm{m}$ in thickness were mounted on membrane slides (Zeiss, Cat. 1.0 PEN 415190-9041) and were deparaffinized and stained for 3-5 seconds in 0.1\% Toluidine Blue Solution (SIGMA, \#T-0394). Laser capture microdissection (LCM) was performed using a type P-MB device (Carl Zeiss MicroImaging). From each section, areas of approximately 500,000 $\mathrm{mm}^{2}$ (roughly 100,000 cells) covering tumor were collected in adhesive caps 200 (Zeiss, Cat. 415190-9181-000). Tissue fragments were stored at $-80^{\circ} \mathrm{C}$ until use. Protein digestion with trypsin was done, followed by purification with $\mathrm{C} 18$ spin columns prior to analysis by mass spectrometry. Mass spectrometry data acquisition was done at NU Proteomics core facility. In brief, peptides were analyzed by LC-MS/MS using a Dionex UltiMate 3000 Rapid Separation nanoLC and a Q Exactive ${ }^{\mathrm{TM}}$ HF Hybrid Quadrupole-Orbitrap ${ }^{\mathrm{TM}}$ Mass Spectrometer (Thermo Fisher Scientific Inc, San Jose, CA). Peptides were separated on a 120-min analytical gradient from $5 \% \mathrm{ACN} / 0.1 \% \mathrm{FA}$ to $30 \% \mathrm{ACN} / 0.1 \% \mathrm{FA}$. The top 15 most abundant precursor ions in each MS1 scan were selected for fragmentation. Proteins were identified from the tandem mass spectra extracted by Xcalibur version 4.0. MS/MS spectra were searched against the SwissProt Homo sapiens database using Mascot search engine (Matrix Science, London, UK; version 2.5.1). The search result was visualized by Scaffold (Proteome Software, INC., Portland, OR). 
Immunohistochemistry (IHC). TMAs were cut at 5-um thickness and deparaffinized. Antigen retrieval was performed at $95^{\circ} \mathrm{C}$ for 30 minutes followed by blocking with HRP solution. Slides were then incubated with antibodies for: AZGP1 (Atlas Antibodies, HPA012582, 1:500), Lipocalin-1 (Elabscience, E-AB-33489, 1:200), Albumin (Abcam, EPSISR1, 1:5,000), S100A7 (Novus Biologicals, NBP1-87205, 1:100), S100A8 (Mybiosource, CF-145, 1:400) and S100A9 (Cell Signaling Technology, D5O6O, 1:150) followed by HRP-conjugated secondary antibody and DAB staining. IHC staining in tumor for AZGP1 (cytoplasmic), Lipocalin-1 (cytoplasmic), Albumin (cytoplasmic), S100A7 (cytoplasmic and nuclear), S100A8 (cytoplasmic and nuclear) and S100A9 (cytoplasmic) were scored by a pathologist (KP) (Supplemental Fig. 1) on a scale from one to three, with one being the weakest staining and three being the strongest staining. All slides were digitized using Nanozoomer 2.0 (Hamamatsu).

TCGA data extraction. We evaluated HNSCC patient clinical and RNA-seq data extracted from TCGA Research Network (http://cancergenome.nih.gov/) and the GEO (https://www.ncbi.nlm.nih.gov/geo/). The TCGA cohort was comprised of 515 patients with clinical and genomic data. For some analysis, RNA-seq data was extracted from HNSCC patients from the following coded sites: 'oropharynx,' 'tonsil' and 'base of tongue'. Preprocessed and normalized htseq count data for AZGP1 and LCN1 gene expression was performed by aligning reads with STAR and Tophat 28,56 . Detection of HPV status and integration sites was performed by realigning data to the HPV genome as previously described ${ }^{28,57}$. AZGP1 RNAseq data for integrated and episomal HPV-positive OPSCC cases were obtained from: https://www.ncbi.nlm.nih.gov/geo/query/acc.cgi?acc=GSE74956. All downloaded data was subsequently normalized using the DESeq2R package ${ }^{58}$.

Statistical methods. Comparisons between elemental concentrations were calculated using paired T tests. The relationship between HPV status and AZGP1/Lipocalin-1/Albumin/S100A7/S100A8/ S100A9 IHC scores were determined by linear regression and Pearson correlation. The relationship between $\log _{2}$-transformed AZGP1 mRNA counts and HPV status was performed by Wilcoxon rank test. The Kaplan-Meier method was used for survival outcomes. The primary endpoint in survival outcomes for cases from NMH was post-treatment recurrence-free survival (RFS) starting at the date of diagnosis and defined as the absence of locoregional or distant recurrence or disease-related death. In HNSCC TCGA survival analysis, the primary endpoint was overall survival. Patient survival trends were tested by log-rank. Multivariable parametric a AFT models were performed to estimate associations between protein expression levels and survival outcomes. Covariate adjustments included: smoking status, age, sex and treatment with surgery only, radiation only, chemoradiation only, surgery followed by radiation or surgery followed by chemoradiation. $P$ values $\leq 0.05$ were considered statistically significant. All statistical analyses were performed in Prism (GraphPad Software) and R (v3.4).

\section{Data availability}

The datasets generated during and/or analysed during the current study are available from the corresponding author on reasonable request.

Received: 19 May 2019; Accepted: 27 October 2019;

Published online: 18 November 2019

\section{References}

1. Sturgis, E. M. \& Cinciripini, P. M. Trends in head and neck cancer incidence in relation to smoking prevalence: an emerging epidemic of human papillomavirus-associated cancers? Cancer 110, 1429-1435 (2007).

2. Jemal, A. et al. Annual Report to the Nation on the Status of Cancer, 1975-2009, featuring the burden and trends in human papillomavirus(HPV)-associated cancers and HPV vaccination coverage levels. J Natl Cancer Inst 105, 175-201 (2013).

3. Blomberg, M., Nielsen, A., Munk, C. \& Kjaer, S. K. Trends in head and neck cancer incidence in Denmark, 1978-2007: focus on human papillomavirus associated sites. Int J Cancer 129, 733-741 (2011).

4. Hajek, M. et al. TRAF3/CYLD mutations identify a distinct subset of human papillomavirus-associated head and neck squamous cell carcinoma. Cancer 123, 1778-1790 (2017).

5. Poropatich, K. et al. Peritumoral cuffing by T-cell tumor-infiltrating lymphocytes distinguishes HPV-related oropharyngeal squamous cell carcinoma from oral cavity squamous cell carcinoma. J Oral Pathol Med (2017).

6. Poropatich K et al. Comprehensive T cell immunophenotyping and next generation sequencing from HPV-positive and -negative head and neck squamous cell carcinomas. J Pathol (2017).

7. Koenigs, M.B. et al. Association of Estrogen Receptor Alpha Expression With Survival in Oropharyngeal Cancer Following Chemoradiation Therapy. J Natl Cancer Inst (2019).

8. Chen, K. M. et al. IGSF4 methylation as an independent marker of human papillomavirus-positive oropharyngeal squamous cell carcinoma. JAMA Otolaryngol Head Neck Surg 141, 257-263 (2015).

9. Schwartz, M. K. Role of trace elements in cancer. Cancer research 35, 3481-3487 (1975).

10. Hordyjewska, A., Popiolek, L. \& Kocot, J. The many "faces" of copper in medicine and treatment. Biometals 27, 611-621 (2014),

11. Labib, H. A., Hassanein, M. \& Etewa, R. L. Serum copper is a simple but valuable prognostic marker in B-cell chronic lymphocytic leukemia. Int J Hematol 100, 575-581 (2014).

12. Geraki, K., Farquharson, M. J. \& Bradley, D. A. Concentrations of Fe, $\mathrm{Cu}$ and $\mathrm{Zn}$ in breast tissue: a synchrotron XRF study. Phys Med Biol 47, 2327-2339 (2002).

13. Margalioth, E. J., Schenker, J. G. \& Chevion, M. Copper and zinc levels in normal and malignant tissues. Cancer 52, 868-872 (1983).

14. Santoliquido, P. M., Southwick, H. W. \& Olwin, J. H. Trace metal levels in cancer of the breast. Surg Gynecol Obstet 142, 65-70 (1976).

15. Ho, E. \& Song, Y. Zinc and prostatic cancer. Curr Opin Clin Nutr Metab Care 12, 640-645 (2009).

16. Franklin, R. B. et al. hZIP1 zinc uptake transporter down regulation and zinc depletion in prostate cancer. Mol Cancer 4, 32 (2005).

17. Lightman, A., Brandes, J. M., Binur, N., Drugan, A. \& Zinder, O. Use of the serum copper/zinc ratio in the differential diagnosis of ovarian malignancy. Clin Chem 32, 101-103 (1986).

18. Papouli, E., Defais, M. \& Larminat, F. Overexpression of metallothionein-II sensitizes rodent cells to apoptosis induced by DNA cross-linking agent through inhibition of NF-kappa B activation. J Biol Chem 277, 4764-4769 (2002). 
19. Arriaga, J. M., Greco, A., Mordoh, J. \& Bianchini, M. Metallothionein $1 G$ and zinc sensitize human colorectal cancer cells to chemotherapy. Mol Cancer Ther 13, 1369-1381 (2014).

20. Xue, Y. N. et al. Zinc promotes prostate cancer cell chemosensitivity to paclitaxel by inhibiting epithelial-mesenchymal transition and inducing apoptosis. Prostate 79, 647-656 (2019).

21. Puca, R. et al. Restoring p53 active conformation by zinc increases the response of mutant p53 tumor cells to anticancer drugs. Cell Cycle 10, 1679-1689 (2011).

22. Puca, R. et al. Reversible dysfunction of wild-type $\mathrm{p} 53$ following homeodomain-interacting protein kinase-2 knockdown. Cancer research $68,3707-3714(2008)$

23. Lu, S. et al. Radiosensitization of clioquinol and zinc in human cancer cell lines. BMC Cancer 18, 448 (2018).

24. Jin, Q. et al. Preserving elemental content in adherent mammalian cells for analysis by synchrotron-based $\mathrm{x}$-ray fluorescence microscopy. J Microsc 265, 81-93 (2017).

25. Melsheimer, P., Vinokurova, S., Wentzensen, N., Bastert, G. \& von Knebel Doeberitz, M. DNA aneuploidy and integration of human papillomavirus type $16 \mathrm{e} 6 / \mathrm{e} 7$ oncogenes in intraepithelial neoplasia and invasive squamous cell carcinoma of the cervix uteri. Clin Cancer Res 10, 3059-3063 (2004).

26. Liu, J. et al. AZGP1 inhibits soft tissue sarcoma cells invasion and migration. BMC Cancer 18, 89 (2018).

27. Kong, B. et al. AZGP1 is a tumor suppressor in pancreatic cancer inducing mesenchymal-to-epithelial transdifferentiation by inhibiting TGF-beta-mediated ERK signaling. Oncogene 29, 5146-5158 (2010).

28. Koneva, L. A. et al. HPV Integration in HNSCC Correlates with Survival Outcomes, Immune Response Signatures, and Candidate Drivers. Mol Cancer Res 16, 90-102 (2018).

29. Finney, L. et al. X-ray fluorescence microscopy reveals large-scale relocalization and extracellular translocation of cellular copper during angiogenesis. Proc Natl Acad Sci USA 104, 2247-2252 (2007).

30. Habib, F. K., Mason, M. K., Smith, P. H. \& Stitch, S. R. Cancer of the prostate: early diagnosis by zinc and hormone analysis? $\mathrm{Br} \mathrm{J}$ Cancer 39, 700-704 (1979).

31. Nardinocchi, L. et al. Zinc downregulates HIF-1alpha and inhibits its activity in tumor cells in vitro and in vivo. PLoS One 5, e15048 (2010).

32. Buntzel, J. et al. Zinc concentrations in serum during head and neck cancer progression. Anticancer Res 27, 1941-1943 (2007).

33. Timar, J. et al. Neoadjuvant immunotherapy of oral squamous cell carcinoma modulates intratumoral CD4/CD8 ratio and tumor microenvironment: a multicenter phase II clinical trial. Journal of clinical oncology: official journal of the American Society of Clinical Oncology 23, 3421-3432 (2005)

34. Eide, D. J. Zinc transporters and the cellular trafficking of zinc. Biochim Biophys Acta 1763, 711-722 (2006).

35. Hassan, M. I., Waheed, A., Yadav, S., Singh, T. P. \& Ahmad, F. Zinc alpha 2-glycoprotein: a multidisciplinary protein. Mol Cancer Res 6, 892-906 (2008).

36. Brysk, M. M. et al. Zinc-alpha2-glycoprotein expression as a marker of differentiation in human oral tumors. Cancer Lett 137, $117-120(1999)$

37. Brysk, M. M. et al. Gene expression of zinc-alpha 2-glycoprotein in normal human epidermal and buccal epithelia. In Vivo 11, 271-274 (1997).

38. Klymenko, T., et al. RNASeq analysis of differentiated keratinocytes reveals a massive response to late events during human papillomavirus type 16 infection, including loss of epithelial barrier function. J Virol (2017).

39. Kumar, A. A. et al. Strong and weak zinc binding sites in human zinc-alpha2-glycoprotein. FEBS Lett 587, 3949-3954 (2013).

40. Zahid, H. et al. Zinc-induced oligomerization of zinc alpha2 glycoprotein reveals multiple fatty acid-binding sites. Biochem J 473, 43-54 (2016).

41. Huang, C. Y. et al. Decreased expression of AZGP1 is associated with poor prognosis in primary gastric cancer. PLoS One 8, e69155 (2013).

42. Parris, T. Z. et al. Additive effect of the AZGP1, PIP, S100A8 and UBE2C molecular biomarkers improves outcome prediction in breast carcinoma. Int J Cancer 134, 1617-1629 (2014).

43. Burdelski, C. et al. Reduced AZGP1 expression is an independent predictor of early PSA recurrence and associated with ERG-fusion positive and PTEN deleted prostate cancers. Int J Cancer 138, 1199-1206 (2016).

44. Yip, P. Y. et al. Low AZGP1 expression predicts for recurrence in margin-positive, localized prostate cancer. Prostate 71, 1638-1645 (2011).

45. Tang, H. et al. Reduction of AZGP1 predicts poor prognosis in esophageal squamous cell carcinoma patients in Northern China. Onco Targets Ther 10, 85-94 (2017).

46. Hale, L. P., Price, D. T., Sanchez, L. M., Demark-Wahnefried, W. \& Madden, J. F. Zinc alpha-2-glycoprotein is expressed by malignant prostatic epithelium and may serve as a potential serum marker for prostate cancer. Clin Cancer Res 7, 846-853 (2001).

47. Lamberti, C., Morrissey, L. C., Grossman, S. R. \& Androphy, E. J. Transcriptional activation by the papillomavirus E6 zinc finger oncoprotein. EMBO J 9, 1907-1913 (1990).

48. Howie, H. L., Katzenellenbogen, R. A. \& Galloway, D. A. Papillomavirus E6 proteins. Virology 384, 324-334 (2009).

49. Tomaic, V. Functional Roles of E6 and E7 Oncoproteins in HPV-Induced Malignancies at Diverse Anatomical Sites. Cancers (Basel) 8 (2016).

50. Lazarczyk, M. et al. Regulation of cellular zinc balance as a potential mechanism of EVER-mediated protection against pathogenesis by cutaneous oncogenic human papillomaviruses. J Exp Med 205, 35-42 (2008).

51. Lewis, J. S. Jr. et al. Human Papillomavirus Testing in Head and Neck Carcinomas: Guideline From the College of American Pathologists. Archives of pathology \& laboratory medicine 142, 559-597 (2018).

52. Arora, H. C. et al. Nanocarriers enhance Doxorubicin uptake in drug-resistant ovarian cancer cells. Cancer research 72, 769-778 (2012).

53. Yuan, Y. et al. Interrogation of EGFR Targeted Uptake of TiO2 Nanoconjugates by X-ray Fluorescence Microscopy. AIP Conf Proc 1365, 423-426 (2011).

54. Nietzold, T. et al. Quantifying X-Ray Fluorescence Data Using MAPS. J Vis Exp (2018).

55. Paunesku, T. et al. X-ray fluorescence microscopy for investigation of archival tissues. Health Phys 103, 181-186 (2012).

56. Atlas, N. Cancer Genome Comprehensive genomic characterization of head and neck squamous cell carcinomas. Nature 517, 576-582 (2015).

57. Zhang, Y. et al. Subtypes of HPV-Positive Head and Neck Cancers Are Associated with HPV Characteristics, Copy Number Alterations, PIK3CA Mutation, and Pathway Signatures. Clin Cancer Res 22, 4735-4745 (2016).

58. Love, M. I., Huber, W. \& Anders, S. Moderated estimation of fold change and dispersion for RNA-seq data with DESeq. 2. Genome Biol 15, 550 (2014).

\section{Acknowledgements}

The research was funded by the Family of Walter Neumann and the 2018 Northwestern Lurie Cancer Center Research Innovation Challenge Award (supported by the IDP Foundation and the Sherman Fairchild Foundation). Work at the Advanced Photon Source at Argonne National Laboratory was supported by the U.S. Department of Energy, Office of Science, Office of Basic Energy Sciences contract DE-AC02-06CH11357. This 
work was also supported by the Northwestern University Pathology Core Facility and a Cancer Center Support Grant (NCI CA060553) with help from Bernice Frederick, Bella Shmaltsuyeva and Shanshan Zhang Proteomics services were performed by the Northwestern Proteomics Core Facility, generously supported by NCI CCSG P30 CA060553 awarded to the Robert H Lurie Comprehensive Cancer Center and the National Resource for Translational and Developmental Proteomics supported by P41 GM108569; authors would especially like to thank the facility director Dr. Young Ah Goo for her assistance with proteomics analysis. Additional thanks are extended to Germaine Elbohy for her assistance with clinical outcome data. Finally, we are grateful to Tiffany Ge for her help with protein zinc content queries.

\section{Author contributions}

K.P., T.P., G.E.W. and B.B.M. helped conceive of the study. K.P., T.P., H.F., S.C., B.L., O.A., E.M., K.B., M.B.W., B.W., D.G. and M.S. carried out data acquisition and data analysis. K.P., P.D., B.B.M., M.A. and G.E. collected clinical information for survival analysis. K.P., A.Z., B.W. and A.R. performed statistical analysis. K.P. drafted the manuscript. All authors read and approved the final manuscript.

\section{Competing interests}

The authors declare no competing interests.

\section{Additional information}

Supplementary information is available for this paper at https://doi.org/10.1038/s41598-019-53268-1.

Correspondence and requests for materials should be addressed to K.P.

Reprints and permissions information is available at www.nature.com/reprints.

Publisher's note Springer Nature remains neutral with regard to jurisdictional claims in published maps and institutional affiliations.

(c) (i) Open Access This article is licensed under a Creative Commons Attribution 4.0 International License, which permits use, sharing, adaptation, distribution and reproduction in any medium or format, as long as you give appropriate credit to the original author(s) and the source, provide a link to the Creative Commons license, and indicate if changes were made. The images or other third party material in this article are included in the article's Creative Commons license, unless indicated otherwise in a credit line to the material. If material is not included in the article's Creative Commons license and your intended use is not permitted by statutory regulation or exceeds the permitted use, you will need to obtain permission directly from the copyright holder. To view a copy of this license, visit http://creativecommons.org/licenses/by/4.0/.

(C) The Author(s) 2019 